Patrick Müller

Stephan Rosenkranz

Oliver Adam

Florian Custodis

Michael Böhm

\section{Clinical trial updates and hotline sessions presented at the European Society of Cardiology Congress 2008}

Received: 18 September 2008

Accepted: 19 September 2008

Published online: 30 October 2008
Dr. med. P. Müller, MD ( $ه)$

S. Rosenkranz $\cdot$ O. Adam

F. Custodis · M. Böhm

Klinik für Innere Medizin III

Kardiologie, Angiologie und Internistische Intensivmedizin

Universitätsklinikum des Saarlandes

Kirrberger Straße

66421 Homburg/Saar, Germany

E-Mail: patrick-mueller@web.de
Abstract This article summarizes the results of a number of clinical trials in the field of cardiovascular medicine which were presented during the Hotline and Clinical Trial Update Sessions at the annual meeting of the European Society of Cardiology, held in Munich, Germany, from 30th August to 3rd September 2008. The data were presented by leading experts in the field with relevant positions in the trials. It is important to note that unpublished reports should be considered as preliminary data, as the analysis may change in the final publications. The comprehensive summaries have been generated from the oral presentation and the webcasts of the European Society of Cardiology and should provide the readers with the most comprehensive information on diagnostic and therapeutic developments in cardiovascular medicine.

Key words PIHRATE - HORIZONS AMI - regent - 3T/2R - APPRAISE-I - SYNTAX - CARDia - IBIS II - DECREASE III - BEAUTIFUL - TIME-CHF - TIMIC - ONTARGET - TRANSCEND - GISSIHF - ATHENA - SEAS

Abbreviations 3-VD: Three vessel disease, ACS: Acute coronary syndrome, ACE-I: Angiotensin converting enzyme inhibitor, AF: Atrial fibrillation, AFL: Atrial flutter, BP: Blood pressure, bpm: Beats per minute, CABG: Coronary artery bypass grafting, CAD: Coronary artery disease, CHF: Congestive heart failure, CI: Confidence interval, CRP: $C$-reactive protein, DES: Drug eluting stents, ECG: Electrocardiogram, ESC: European Society of Cardiology, GPIIb/IIIa: Glycoprotein IIb IIIa, HR: Hazard ratio, LDL: Low density lipoprotein, LM: Left main coronary artery, LVEF: Left ventricular ejection fraction, MACE: Major adverse cardiovascular events, MBG: Myocardial blush grade, MI: Myocardial infarction, MRI: Magnetic resonance imaging, NNT: Number needed to treat, NS: Not significant, NSTE-ACS: Non-ST-segment elevation acute coronary syndrome, NSTEMI: Non-ST-segment elevation myocardial infarction, NYHA: New York Heart Association, OR: Odds ratio, PCI: Percutaneous coronary intervention, RR: Relative risk, STEMI: ST-segment elevation myocardial infarction, STR: ST segment elevation resolution, TVR: Target vessel revascularization, TIMI: Thrombolysis in myocardial infarction group, UFH: Unfractionated heparin 


\section{Acute coronary syndrome: interventions and concomitant treatments}

\section{Polish-Italian-Hungarian randomized thrombectomy trial (PIHRATE trial): D. Dudek (Krakow, Poland)}

The PIHRATE trial is an international prospective multicentre non-blinded randomized study comparing thrombectomy in addition to PCI versus conventional PCI (balloon predilatation followed by direct stenting) in STEMI. The trial investigates whether simple thrombus aspiration followed by direct primary percutaneous coronary intervention (PPCI) may reduce distal embolization and improve myocardial reperfusion, because in two-thirds of patients after conventional PPCI (stent implantation and even with GP IIb/IIIa inhibitors) is an impaired microcirculatory perfusion observed (MBG grade 0$2)$, accompanied by partial $(30 \%-70 \%)$ or no $(<30 \%)$ ST-segment elevation resolution (STR) in ECG) These patients have increased early and late mortality, and a higher incidence of heart failure.

Hundred and ninety six patients with STEMI $<6 \mathrm{~h}$ and occluded infarct related artery (TIMI grade flow $0-1$ ) at baseline angiography were randomized to two groups: 100 patients in the thrombectomy group (A) and 96 patients in the control group (B) at 10 PPCI centers in Poland, Italy and Hungary. The primary end point of the trial was STR in the ECG after PPCI. Secondary end points included: direct stenting rate, final TIMI grade 3 flow, corrected TIMI Frame count (cTFC), final MBG grade 3, periprocedural angiographic complications, combination of STR $\geq 70 \%$ and MBG 3 (optimal myocardial reperfusion), and in-hospital major adverse cardiac events (MACE). Aspiration thrombectomy was successful in $91 \%$ of the cases. The study groups did not differ in the stenting rate, stent length, or maximal stent deployment pressure, except for the direct stenting rate which was higher in the thrombectomy group (75\% vs. $5.2 \%, P<0.0001)$. STR $\geq 70 \%$ was significantly better in the group with thrombectomy (41.4 vs. $25.9, P=0.037$ ) directly after the procedure but at $60 \mathrm{~min}$ after procedure, only a trend was observed (53.7\% vs. $35.1 \%, P=0.29$ ). There was no difference in final TIMI grade 3 flow $(87.8 \%$ vs. $81.3 \%$, $P=0.36$, group A vs. group B) but the final cTFC $(28 \pm 15$ vs. $33 \pm 19, P=0.027)$ and $M B G$ rates (MBG grade $3,76.1 \%$ vs. $57.8 \%, P=0.026$ ) were significantly better in group A. Also the combined angiographic end point of TIMI grade 3 flow + MBG grade $3(72.7 \%$ vs. $54.2 \%, P=0.012)$ as well as STR $\geq 70 \%+$ MBG grade $3(35.1 \%$ vs. $11.8 \%, P<0.0001)$ was significantly better in the thrombectomy group but death $(4.0 \%$ vs. $3.1 \%)$ and myocardial reinfarction $(1.0 \%$ vs. $3.1 \%, P=\mathrm{NS})$ rates were similar after 6 months follow up.

In conclusion, in PIHRATE the strategy of aspiration thrombectomy compared with primary PCI failed to reduce ST-resolution $60 \mathrm{~min}$ after PCI in STEMI patients presenting within $6 \mathrm{~h}$ after symptom onset, although it did improve myocardial blush grade of 3 . There was no obvious benefit with clinical endpoints at 6 months of follow-up. PIHRATE thereby confirms the lack of impact of aspiration thrombectomy on mortality and re-myocardial infarction. Only one large scale trial (the TAPAS trial) demonstrated improved myocardial flow and perfusion which translated into less cardiac mortality and less combined death/re-MI.

\section{Impact of major adverse cardiac events and major bleeding on overall mortality in patients with STEMI: the HORIZONS AMI trial: R. Mehran (New York, USA)}

In HORIZONS AMI more than 3,600 patients with STEMI (symptom onset $<12 \mathrm{~h}$ ) from 123 centers in 11 countries were randomized 1:1 to unfractionated heparin (UFH, $60 \mathrm{U} / \mathrm{kg} \mathrm{IV}$ ), with subsequent boluses titrated by nomogram to activated clotting time of 200-250 s, plus a GP IIb/IIIa inhibitor (abciximab or eptifibatide), or to bivalirudin monotherapy $(0.75 \mathrm{mg} / \mathrm{kg}$ bolus; infusion $1.75 \mathrm{mg} / \mathrm{kg}$ per $\mathrm{h})$, stopped at the end of the procedure, plus provisional GP IIb/IIIa inhibitors for large thrombus or refractory no-flow [1]. Only $7.2 \%$ of patients in the bivalirudin-treated group also received GP IIb/IIIa inhibitors in the catheter laboratory. The trial was designed to test for noninferiority and superiority of bivalirudin compared to the UFH-GP IIb/IIIa-inhibitor strategy. Primary end points were net adverse clinical events and major bleeding At 30 days bivalirudin monotherapy led to a $24 \%$ reduction in net adverse clinical events and a $40 \%$ reduction in major bleeding. MACE, defined as all-cause death, reinfarction, ischemic target vessel revascularization (TVR), or stroke, were no different between the two groups (Table 1). There was no significant differences between any of the individual event rates that made up the MACE end point, with the exception of cardiac mortality, which was significantly reduced in patients in the bivalirudin arm of the study (Table 2).

To determine the impact of major bleeding and/or MACE, the investigators developed a multivariable Cox model with five significant baseline predictor 
Table 1 HORIZONS AMI trial: major outcomes

\begin{tabular}{|c|c|c|c|c|c|c|}
\hline End point & $\begin{array}{l}\text { UFH-GP Ilb/IIla } \\
\text { inhibitors (\%) }\end{array}$ & Bivalirudin (\%) & $\begin{array}{l}\text { Absolute } \\
\text { difference (\%) }\end{array}$ & $\begin{array}{l}\text { Relative risk } \\
\text { reduction (\%) }\end{array}$ & $P$, noninferiority & $P$, superiority \\
\hline NACE & 12.1 & 9.2 & -2.9 & 24 & $\leq 0.0001$ & 0.006 \\
\hline Major bleeding & 8.3 & 4.9 & -3.3 & 40 & $\leq 0.0001$ & $\leq 0.0001$ \\
\hline MACE & 5.5 & 5.4 & - & - & - & NS \\
\hline
\end{tabular}

NACE net adverse clinical events

Table 2 HORIZONS AMI trial: death at 30 days

\begin{tabular}{llll}
\hline End point & UFH-GP IIb/IIla inhibitors (\%) & Bivalirudin (\%) & $P$ \\
\hline All death & 3.1 & 2.1 & 0.058 \\
Cardiac death & 2.9 & 1.8 & 0.035 \\
\hline
\end{tabular}

variables of mortality within 30 days of presentation with STEMI. The main event endpoints from the trial (including the components of MACE and major bleeding) were then added to the model as time updated covariates. Within 30 days of randomization, there were 93 deaths; 26 following a non CABG-related major bleed in 238 patients, 10 deaths following a reinfarction in 65 patients, 9 following 82 ischemic TVRs, and 3 following 22 strokes. In the fully adjusted models, time updated reinfarction and non-CABG major bleeding were significantly associated with mortality. Ischemic TVR and stroke were not significantly associated with mortality within the first 30 days.

In conclusion, in HORIZON AMI-after accounting for baseline predictors-both reinfarction and major bleeding have a significant impact on mortality in the first 30 days after presenting with STEMI. While the hazard ratio for reinfarction is nominally higher, there are more deaths attributable to major bleeding as compared with a reinfarction within the first 30 days in this population. As most drugs which reduce ischemia also increase bleeding, the offsetting impact of adverse ischemic and hemorrhagic events must be carefully examined in future trials.

\section{Myocardial regeneration by intracoronary infusion of selected population of stem cells in acute myocardial infarction (REGENT) randomized multicenter trial: M. Tendera (Katowice, Poland)}

REGENT is a multicenter, randomized trial comparing the intracoronary infusion of bone marrow-derived unselected mononuclear cells and selected CD34 + CXCR4 + cells against a control group in 200 patients with acute myocardial infarction and reduced LVEF $\leq 40 \%$.

REGENT is after REPAIR-AMI the second-largest trial using bone marrow-derived cells in patients with acute MI and the first large trial for head-to-head comparison of selected and unselected cells. The population of CD34 + CXCR4 + cells is enriched in cardiac committed progenitor cells, but so far, it has not been used in a clinical trial.

Change in LVEF and volumes measured by MRI before and 6 months after the procedure served as the primary endpoint. In patients receiving both selected and unselected bone marrow cells, LVEF increased significantly in comparison to baseline values by $3 \%$. However, the increase in both treatment groups was not significantly higher in comparison to the control group. The changes in left ventricular volumes were also comparable in all groups. Baseline LVEF was an independent predictor of significant increase of LVEF. At 6 months clinical follow-up, major cardiovascular event rate was low, and no difference between the groups was observed. Intracoronary infusion of unselected and selected bone marrow cells proved to be safe and feasible.

These results suggest that in patients with severely depressed LVEF, administration of a relatively small number of CD34 + CXCR4 + cells is safe and feasible and can have the same effect as an infusion of a larger number of unselected mononuclear cells. However despite trends toward improvement of LVEF in patients with severely depressed baseline LVEF receiving either selected or unselected bone marrow cells there was no significance observed.

\section{Main results of the tailoring treatment with tirofiban in patients showing resistance to aspirin and/or resistance to clopidogrel study (3T/2R): M. Valgimigli (Ferrara, Italy)}

The goal of the study was to evaluate whether tirofiban would reduce the incidence of MI after PCI among poor responders to aspirin and/or clopidogrel.

There were 263 patients enrolled in the study who were poor responders to aspirin and/or clopidogrel, 
based on point-of-care assays, who underwent elective coronary angioplasty at ten European sites for stable or low-risk unstable coronary artery disease. Patients were randomly assigned in a double blind manner to receive either a high-dose bolus tirofiban or placebo on top of standard aspirin and clopidogrel therapy.

The primary end point was the occurrence of periprocedural myocardial infarction, as defined by an increase in Troponin $\mathrm{I}$ or $\mathrm{T}$ greater than three times the upper limit of normal within $48 \mathrm{~h}$. Tirofiban reduced MACE at 30 days compared to placebo (21\% vs. $37 \% ; P=0.0065)$. There were no major bleedings in either group.

The authors interpreted their findings as a proof of concept for a new treatment strategy in patients with an increased risk of thrombotic events due to nonresponsiveness of patients to standard oral antiplatelets such as aspirin or clopidogrel. However the discussant criticized that the study did not use all tests available for determining aspirin or clopidogrel resistance and that there was no "non-resistant" group of patients as a control group. Since the use of GPIIb/IIIa is already recommended in the ESC guidelines in complex lesions as well as a pre-treatment before diagnostic angiography and possible PCI within $48 \mathrm{~h}$ in high risk patients, it remains to be doubted if the study will change the already established guidelines.

\section{Safety of the factor Xa inhibitor, apixaban, in combination with antiplatelet therapy after acute coronary syndrome: results of the APPRAISE-I dose guiding trial: J. Alexander (Durham, USA)}

APPRAISE- 1 is a phase II, dose-ranging study of the oral, direct factor Xa inhibitor, apixaban. Thousand seven hundred and fifteen patients with recent STEMI or NSTE-ACS were randomized to placebo $(n=611)$ or one of four doses of apixaban $2.5 \mathrm{mg}$ BID $(n=317), \quad 10 \mathrm{mg} \quad$ QD $\quad(n=318), \quad 10 \mathrm{mg} \quad$ BID $(n=248)$, or $20 \mathrm{mg}$ QD $(n=221)$ for 6 months. All patients received aspirin. Use of clopidogrel was at the discretion of the treating physician.

The primary outcome of the study was major or clinically relevant non-major bleeding by International Society of Thrombosis and Hemostasis (ISTH) criteria. Secondary outcomes were cardiovascular death, myocardial infarction, severe recurrent ischemia or stroke.

The $10 \mathrm{mg}$ BID and $20 \mathrm{mg}$ QD apixaban arms were discontinued early due to excess bleeding. Results from the placebo and the two lower dose apixaban arms were presented.

In comparism to placebo (3.0\%), apixaban $2.5 \mathrm{mg}$ BID showed a trend (5.7\%, HR 1.78, 95\% CI 0.91-3.48,
$P=0.09)$ towards a dose dependent increase in ISTH major or clinically relevant non-major bleeding, whereas apixaban $10 \mathrm{mg}$ QD reached this primary outcome in a significant manner (7.9\%, HR 2.45, 95\% CI 1.31-4.61, $P=0.005)$. Additionally, both evaluated doses of apixaban resulted in non-signifcant trends (2.5 mg BID: 7.6\%, HR 0.73, 95\% CI 0.44-1.19, $P=0.21 ; 10$ mg QD: $6.0 \%$, HR 0.61, 95\% CI 0.35-1.04, $P=0.07)$ toward a reduction in clinically important recurrent ischemic events compared to placebo especially in patients not taking clopidgrel.

In conclusion it was discussed that it remains unclear what the net clinical effect of apixaban on top of aspirin or clopidogrel is since the adverse effects regarding bleeding risk have to be weighted against the trend towards a clinical benefit concerning reduction of ischemic events.

\section{The synergy between percutanous coronary intervention and cardiac surgery (SYNTAX) study: P. Serruys (Rotterdam, The Netherlands) and W. Mohr (Leipzig, Germany)}

Since interventional revascularization strategies have experienced major developments during the last years, PCI with DES is challenging CABG as the gold standard for treatment of three-vessel and left main coronary disease. Conducted at 62 European sites and 23 sites in the US the Syntax trial is the first randomised controlled clinical trial to compare PCI using drug-eluting stents (TAXUS) to coronary artery bypass grafting $(\mathrm{CABG})$ in patients with left main (LM) or/and three vessel disease (3-VD). The leading hypothesis of the trial was that DES-PCI would be non-inferior to CABG in the management of patients with 3-vessel disease and/or left main disease.

All patients were assessed by a local multidisciplinary team including interventional cardiologists and cardiac surgeons. If both, the cardiologist and the surgeon felt they could offer revascularisation (if the anatomy was suitable for both CABG and PCI), patients were randomized in a $1: 1$ fashion to PCI or CABG. The trial randomized 1,800 patients to either CABG $(n=897)$ or PCI $(n=903)$ with the Taxus DES, with a primary end point of 12 -month major adverse cardiac and cerebrovascular events (MACCE), defined as all-cause death, cerebrovascular event, MI and repeat revascularization (PCI and/or CABG). Secondary outcome measures included overall MAC$\mathrm{CE}$ at different follow-up periods, rates of individual end points and quality-of-life and cost-effectiveness measures. Due to the "all comers" study design patients who were ineligible for either CABG or PCI were entered into one of two registries: 1,077 into the CABG registry and 198 into the PCI registry. 
Table 3 SYNTAX trial

\begin{tabular}{lccc}
\hline End point & CABG (\%) & DES (\%) & $P$ \\
\hline MACCE & 12.1 & 17.8 & 0.0015 \\
Death/MI/stroke & 7.7 & 7.6 & 0.98 \\
Revascularization & 5.9 & 13.7 & $<0.0001$ \\
Stroke & 2.2 & 0.6 & 0.003 \\
MI & 3.2 & 4.8 & 0.11 \\
All-cause death & 3.5 & 4.3 & 0.37 \\
\hline
\end{tabular}

Primary endpoint occurrence with CABG vsersus DES-PCI

The incidence of the primary endpoint of major adverse cardiac and cerebrovascular events (MACCE) at 12 months was lower in the CABG arm compared with PCI $(12.1 \%$ vs. $17.8 \%, P=0.0015)$, and did not meet the prespecified non-inferiority threshold for PCI. This was driven predominantly by a significant reduction in the incidence of repeat revascularization in the CABG arm compared with PCI (5.9\% vs. $13.7 \%$, $P=0.0001)$. There was no difference in the incidence of death $(P>0.05)$ or MI between the two arms $(P=0.11)$. The incidence of cerebrovascular accident was significantly higher in the CABG arm $(2.2 \%$ vs. $0.6 \%, P=0.003$ ), whereas the incidence of symptomatic graft occlusion and stent thrombosis was similar between the two arms $(3.4 \%$ vs. $3.3 \%$, $P=0.89$ ) (Table 3).

In a subgroup analysis of the LM patients, the overall 12-month MACCE event rate was similar in CABG and PCI (13.7\% vs. $15.8 \%)$, although patients with LM only ( $8.5 \%$ vs. $7.1 \%)$ and LM + 1-VD $(13.2 \%$ vs. $7.5 \%)$ seemed to do slightly better with PCI. Patients with $\mathrm{LM}+2-\mathrm{VD}(14.4 \%$ vs. $19.8 \%), \mathrm{LM}+3-$ VD (15.4\% vs. $19.4 \%)$, or 3 -VD alone $(11.5 \%$ vs. $19.2 \%)$ seemed to do better with CABG than PCI. When stratified by diabetes status, patients with diabetes had lower 12-month MACCE event rates with CABG than with PCI $(14.2 \%$ vs. $26.0 \%, P=0.0025)$. Non-diabetic patients showed a trend toward a benefit with CABG but it was not statistically significant (11.8\% vs. $15.1 \%, P=0.08)$.

In conclusion the trial demonstrates that in patients with LM disease and/or severe 3-VD, CABG (with the use of at least one arterial graft) appears to be superior to PCI with TAXUS DES. This is the case for reducing 12-month MACCE rates, which were predominantly driven by the need for repeat revascularization in the PCI arm. CABG is, however, associated with a higher risk of CVA at 12 months, compared with PCI. Importantly, there is no difference in the incidence of death, MI, or graft occlusion/ stent thrombosis between the two arms. The largest benefit from CABG seems to be in patients with diabetes mellitus. The results of this trial also suggest that patients with LM only, LM + 1-VD, and nondi- abetics may do as well with both CABG and PCI, although the trial was not powered to study these differences in these subgroups. With respect to those patients included in the registries the authors concluded that in patients who are not eligible for PCI, CABG represents an option and, at least in patients not eligible for $C A B G$, they described PCI as a "viable" option.

\section{Coronary artery revascularization in diabetes. The CARDia trial: A. Kapur (London, Great Britain)}

Since the BARI trial had suggested that CABG improves survival in patients with diabetes compared to angioplasty, this finding has guided practice. Therefore the CARDia trial was designed to test the hypothesis that optimal PCI is not inferior to modern CABG as a revascularisation strategy. Five hundred and ten patients with diabetes and multivessel or complex single vessel disease were randomised to PCI or CABG.

Results after 1 year showed no apparent difference between CABG and PCI in terms of the composite endpoints of death, non fatal MI and non fatal stroke $(10.2 \%$ vs. $11.6 \%, P=0.63)$. Comparison of the individual endpoints of CABG versus PCI showed also no significant difference for death $(3.3 \%$ vs. $3.2 \%$, $P=0.83)$, non fatal MI $(5.7 \%$ vs. $8.4 \%, P=0.25)$ and non fatal stroke $(2.5 \%$ vs. $0.4 \%, P=0.09)$. As expected repeat revascularisation was significantly higher in the PCI group with a rate of $9.9 \%$ versus $2.0 \%$ for CABG. Subgroup analysis comparing CABG and 179 PCI patients who received drug eluting $\mathrm{Cy}-$ pher stents rather than bare metal stents, showed no difference in the composite endpoint of death, non fatal MI and non fatal stroke $(10.2 \%$ vs. $10.1 \%$; $P=0.98$ ). The results of the CARDia trial indicate that diabetic patients with $3-\mathrm{VD}$ or complex 1-VD, but not LM disease, have a similar incidence of death, MI, or stroke at 12 months with either PCI or CABG. However, a higher incidence of repeat revascularization with PCI is found in these patients, even with DES. There is also a higher incidence of stroke in the CABG arm compared with PCI. One of the limitations of this trial is that it may be underpowered to study differences between the two arms, since the event rates used for sample size calculations were significantly different from those observed in the study. Even so, the results of this trial are similar to the SYNTAX trial, which demonstrated better outcomes in diabetic patients with LM disease and/or 3-VD, primarily due to a reduction in the incidence of repeat revascularization.

Long-term follow-up data of the CARDia trial are awaited to assess the durability of these results. 
Table 4 DECREASE III trial

\begin{tabular}{lcclc}
\hline Outcome & $\begin{array}{l}\text { Odds } \\
\text { ratio }\end{array}$ & $95 \% \mathrm{Cl}$ & $\begin{array}{l}\text { Absolute risk } \\
\text { reduction (\%) }\end{array}$ & NNT \\
\hline Myocardial ischemia & 0.53 & $0.32-0.88$ & -8.0 & 13 \\
Nonfatal Ml & 0.55 & $0.24-1.27$ & -2.8 & 36 \\
Cardiovascular death & 0.33 & $0.09-1.22$ & -2.4 & 42 \\
CV death or nonfatal Ml & 0.48 & $0.24-0.95$ & -5.3 & 19 \\
\hline
\end{tabular}

Odds ratios for primary and secondary outcomes (statin patients compared with placebo)

\section{Effects of the direct lipoprotein-associated phospholipase $A_{2}$ inhibitor darapladib on human coronary atherosclerotic plaque: $\mathbf{W}$. Wijns (Aalst, Belgium)}

The IBIS II investigators presented a randomized phase II exploratory study on 330 patients examining the effect of Lp-PLA2 inhibition with darapladib (160 mg daily) after 12 months [2].

Background of the study was that lipoproteinassociated phospholipase $A_{2}\left(\mathrm{Lp}-\mathrm{PLA}_{2}\right)$ is expressed abundantly in the necrotic core of coronary lesions, and products of its enzymatic activity may contribute to inflammation and cell death, rendering plaque vulnerable to rupture.

The study population were patients of at least 18 years of age undergoing catheterization for stable coronary artery disease or an acute coronary syndrome. Background therapy was comparable between groups, with no difference in low-density lipoprotein cholesterol at 12 months. In contrast, Lp-PLA 2 activity was significantly inhibited by $59 \%$ with darapladib $(P<0.001$ vs. placebo $)$. MACE were similar between the groups.

Concerning the two primary endpoints plaque deformability (measured by intravascular ultrasound palpography) and reduction of high sensitivity $C$ reactive protein (hs-CRP), there were no significant differences between darapladib-treated patients and the control group after 12 months (both $P=0.22$ ). In the placebo-treated group, however, necrotic core volume-a secondary endpoint of the study increased significantly $\left(4.5 \pm 17.9 \mathrm{~mm}^{3} ; \quad P=0.009\right)$, whereas darapladib halted this increase $\left(-0.5 \pm 13.9 \mathrm{~mm}^{3}\right.$; $P=0.71$ ), resulting in a significant treatment difference of $-5.2 \mathrm{~mm}^{3}(P=0.012)$. But these intraplaque compositional changes occurred without a significant treatment difference in total atheroma volume $(P=0.95)$.

The investigators concluded from these findings that Lp-PLA ${ }_{2}$ inhibition may represent a novel therapeutic approach. However in the discussion it was criticized that the study did not reach the primary endpoints and that the study design was prominently based on intravascular ultrasound (IVUS)-oriented nonvalidated surrogate end points rather than on better validated surrogate end points, such as carotid intima-media thickness, flow-mediated dilation, or coronary plaque volume, which are known to be better correlated with clinical end points.

\section{Fluvastatin XL use is associated with improved cardiac outcome after major vascular surgery. Results from a randomized placebo controlled trial: DECREASE III: D. Poldermans (Rotterdam, The Netherlands)}

In patients undergoing non-cardiac vascular surgery perioperative cardiac events are the major cause of adverse outcome. Recent retrospective studies suggested a potential beneficial role of statins in the prevention of perioperative myocardial infarction. In the prospective, randomized, placebo controlled DECREASE III study 497 statin-naive patients scheduled to undergo vascular surgery were randomized to a sustained-release formulation of fluvastatin $(n=250)$ $80 \mathrm{mg}$ per day or placebo $(n=247)$ an average of 37 days prior to surgery. The statin was continued for at least the first 30 days after surgery. The primary end point was myocardial ischemia, as assessed by a combination of continuous ECG monitoring in the first $72 \mathrm{~h}$, intermittent troponin- $\mathrm{T}$ measurements and further ECGs until the end of follow-up on day 30. Myocardial ischemia was detected in 74 (14.9\%) patients within 30 days of the initial vascular surgical procedure. A total of $27 / 250(10.9 \%)$ patients allocated to fluvastatin reached the primary endpoint compared to $47 / 247(18.9 \%)$ patients allocated to placebo treatment (OR $0.53 ; 95 \%$ CI $0.32-0.88$ ). Hence, NNT to prevent one patient experiencing myocardial ischemia was 12.5 patients. A total of 18 (3.6\%) patients died within 30 days after surgery of which $12(2.4 \%)$ were attributable to cardiovascular causes. Additionally, 25 (5.0\%) patients experienced a nonfatal myocardial infarction within 30 days after surgery. The combined endpoint of cardiovascular death and nonfatal myocardial infarction was reached in $37 / 497(7.4 \%)$ patients. A total of $12 / 250(4.8 \%)$ patients allocated to fluvastatin therapy reached the combined endpoint, compared to 25/247 (10.1\%) allocated to placebo. Hence, fluvastatin therapy was associated with a $52 \%$ relative reduction in the incidence of cardiovascular death or MI (OR 0.48; 95\% CI 0.24-0.95) (Table 4). The NNT for the composite endpoint of cardiovascular death or nonfatal MI is 18.9 patients. In addition to clinical endpoints the effect of statin treatment on inflammatory markers such as interleukin-6 (IL-6) and CRP was assessed. IL- 
Table 5 BEAUTIFUL trial

\begin{tabular}{llr}
\hline End point & Hazard ratio & $P$ \\
\hline Cardiovascular death & 1.34 & 0.0041 \\
Admission for heart failure & 1.53 & $<0.0001$ \\
Admission for Ml & 1.46 & 0.0066 \\
Coronary revascularization & 1.38 & 0.037 \\
\hline
\end{tabular}

Adjusted hazard ratios for heart rate $>70$ versus $<70 \mathrm{bpm}$

6 levels (5.75 vs. $8.45 \mathrm{pg} / \mathrm{ml}, P<0.001)$ and CRP (4.66 vs. $6.00 \mathrm{mg} / \mathrm{l}, P \leq 0.001)$ were significantly reduced in the fluvastatin-group compared with placebo.

In conclusion, fluvastatin seems a safe choice to improve perioperative outcome in CABG. It remains to be examined if this is a specific fluvastatin effect or rather a group effects of the statins.

\section{Heart failure}

\section{Morbidity-mortality evaluation of the $I_{f}$ inhibitor ivabradine in patients with coronary artery disease and left ventricular dysfunction (BEAUTIFUL): K. Fox (Brompton Hospital, UK)}

Ivabradine is a specific inhibitor of the $I(\mathrm{f})$ current in the sinoatrial node and therefore lowers heart rate, without affecting other aspects of cardiac function. The BEAUTIFUL trial aimed to test whether lowering heart rate with ivabradine reduces cardiovascular death and morbidity in patients with coronary artery disease $\mathrm{CAD}$ and left-ventricular systolic dysfunction (LVEF < 40\%).

BEAUTIFUL, a randomised, double-blind, placebo-controlled, parallel-group trial, enrolled 10.917 patients from 781 centers in 33 countries, and randomized them to $5 \mathrm{mg}$ ivabradine (with the aim of titrating up to $7.5 \mathrm{mg}$ twice per day) or matched placebo, on top of best medical therapy (including high rates of aspirin, ACE-I, and beta blockers). Eighty seven percent of the patients received betablockers in addition to study drugs. The primary endpoint was a composite of cardiovascular death, admission to hospital for acute myocardial infarction, and admission to hospital for new onset or worsening heart failure. The mean heart rate at baseline was $71.6 \pm 9.9 \mathrm{bpm}$. At a median of 19 months of followup, ivabradine reduced heart rate by $6 \pm 0.2 \mathrm{bpm}$ but did not affect the primary composite end point (hazard ratio $1.00,95 \%$ CI $0.91-1.1, P=0.94$ ) [3] In a subanalysis of the placebo group, the BEAUTIFUL investigators demonstrated-as expected [4] but never proven-that elevated resting heart rate at baseline is a marker for subsequent cardiovascular death and morbidity, as patients with heart rates of $70 \mathrm{bpm}$ or greater had increased risk for cardiovascular death $(34 \%, P=0.0041)$, admission to hospital for heart failure $(53 \%, P<0.0001)$, admission to hospital for myocardial infarction $(46 \%, P=0.0066)$, and coronary revascularisation $(38 \%, P=0.037)$ [5] (Table 5). Nevertheless, even in a prespecified subgroup of patients with heart rate of $\geq 70 \mathrm{bpm}$, ivabradine had no effect on the primary composite outcome (HR $0.91,95 \%$ CI $0.81-1.04, P=0.17$ ). It did, however, reduce secondary endpoints such as admission to hospital for fatal and non-fatal myocardial infarction (HR 0.64, 95\% CI 0.49-0.84, $P=0.001$ ) and coronary revascularisation (HR 0.70 , 95\% CI $0.52-0.93, P=0.016$ ), even on top of betablocker therapy [3]. These data on secondary endpoints should be interpreted with caution since the results may be confounded by the fact that the use of beta-blockers was slightly higher in the group with heart rates lower than $70 \mathrm{bpm}(90 \%$ vs. $84 \%)$, which may account for the larger effect of ivabradine among the patients with higher heart rates. Secondly, the large number of secondary endpoints in BEAUTIFUL bears the risk of false-positive results in subgroup analyses. Therefore, the data regarding secondary endpoints warrant careful statistical analysis before any formal conclusions can be drawn.

In summary, ivabradine appears to safely lower heart rate, but while doing so has no effect on cardiac death, need for PCI, or admission to the hospital for heart failure or $\mathrm{MI}$ in patients with stable CAD and LV dysfunction. Nevertheless, it may be useful to reduce the incidence of CAD outcomes in a subgroup of patients who have heart rates $\geq 70 \mathrm{bpm}$. At present, beta-blockers remain the first choice to lower heart rate in patients with heart failure, but ivabradine is likely to become a valuable alternative in patients who do not tolerate beta-blocker therapy. Additional information on the role of ivabradine in CHF patients will be provided by the SHIFT trial [6].

\section{Trial of intensified (BNP-guided) versus standard (symptom-guided) medical therapy in elderly patients with congestive heart failure: TIME-CHF: HP. Brunner-La Rocca (Basel, Switzerland)}

The TIME-CHF study was designed to evaluate the medical management of very old patients ( $\geq 75$ years) with CHF compared with younger patients (6074 years) (Table 6), and to compare an intensified with a standard treatment approach. The rationale behind the study was the fact that the group of patients older than 75 years has been greatly underrepresented in clinical CHF trials, and that the evidence on which the current guidelines are based, 
Table 6 TIME-CHF trial

\begin{tabular}{llll}
\hline Feature & $\begin{array}{l}\text { Age 60-74 } \\
n=210\end{array}$ & $\begin{array}{l}\text { Age }>75 \\
n=289\end{array}$ & $P$ \\
\hline Mean age (year) & 69 & 89 & $<0.001$ \\
Female (\%) & 25 & 41 & $<0.001$ \\
CAD heart-failure etiology (\%) & 49 & 64 & $<0.001$ \\
LVEF (\%) & 28 & 10 & $<0.001$ \\
NYHA class $\geq 3$ (\%) & 66 & 81 & $<0.001$ \\
$\geq 2$ comorbidities (\%) & 64 & 79 & $<0.001$ \\
Creatinine ( $\mu \mathrm{m} / \mathrm{l})$ & 111 & 121 & 0.004 \\
\hline
\end{tabular}

Baseline differences between the younger (age 64-74 years) and elderly (age $>75$ years) patient groups

does not adequately represent this patient population [7].

In TIME-CHF, a prospective single-blinded multicenter trial, 499 patients with symptomatic heart failure ( $\geq$ NYHA class 2 ; LVEF $\leq 45 \%$ ) despite medical treatment who had been hospitalized for CHF within the previous year and had an elevated N-terminal brain-type natriuretic peptide level (NT-proBNP; $\geq$ twice the upper limit of normal), were randomized to natriuretic-peptide-guided or symptom-guided management. Medical treatment strategies followed the European guidelines (including ACE-I, $\mathrm{AT}_{1}$ receptor blockers, beta-blockers, and, for patients in NYHA class 3 or worse, spironolactone or eplerenone) with the aim to reduce symptoms to NYHA class $\leq 2$ (standard) or, additionally, NT-proBNP levels below $400 \mathrm{pg} / \mathrm{ml}$ for patients aged $60-74$ years or below $800 \mathrm{pg} / \mathrm{ml}$ for those $\geq 75$ years (intensified). The primary study end point was hospitalization-free survival at 18 months. Independently of the patient's age, "intensified" therapy guided by NT-proBNP levels led to higher dosages of ACE-I, beta blockers, and other standard CHF medications. Both NTproBNP-guided therapy and standard management significantly reduced NT-proBNP levels from baseline to 6 months to a similar degree. Overall, the HR (95\% CI) of standard therapy vs. natriuretic peptide-guided therapy for the primary end point, 18-month survival free of any hospitalization, was 0.92 (0.73-1.15). Hence, the use of natriuretic-peptide levels rather than symptoms alone to guide medical treatment of CHF failed to influence the primary end point of hospitalization-free survival. The natriuretic-peptidebased approach did however improve survival free from CHF hospitalizations, a secondary end point, among the prospectively defined subgroup of patients $<75$ years. In contrast, patients $\geq 75$ years did not show any outcome difference between the two management strategies. Interestingly, the improvements in quality-of-life scores were less pronounced on intensified therapy as compared to standard symp- tom-guided management in the older patients $(P<0.05)$, whereas they were similar in the younger patients. This finding indicates that intensified medical therapy may cause some harm in the elderly patients who tend to have more co-morbidities than younger patients.

The study indicates that CHF patients $\geq 75$ years respond differently to heart failure therapies compared with younger patients, and furthermore suggests that lessons from the CHF trials on which treatment guidelines are based may not apply to elderly patients with heart failure. As a result, clinical CHF trials targeting older patients are warranted.

\section{Randomized study on the efficacy of immunosuppressive therapy in patients with virus-negative inflammatory cardiomyopathy: A. Frustaci (Rome, Italy)}

There is currently no consensus on the management of patients with chronic inflammatory dilated cardiomyopathy, other than the use of standard heart failure medications. The role of immunosuppressive therapy in these patients, both pediatric and adult, remains controversial. The goal of the single center randomized TIMIC-trial was to evaluate the efficacy of immunosuppressive therapy in adult patients with virus-negative inflammatory cardiomyopathy.

Patients with inflammatory cardiomyopathy who were unresponsive to standard heart failure therapy and were virus negative received either immunosuppressive therapy with prednisone $(1 \mathrm{mg} / \mathrm{kg}$ daily for $1 \mathrm{month}$, followed by $0.33 \mathrm{mg} / \mathrm{kg}$ daily for 5 months), and azathioprine $(2 \mathrm{mg} / \mathrm{kg}$ daily for 6 months), or matching placebo. Eighty five patients were randomized, 43 to the immunosuppressive therapy arm, and 42 to the placebo arm. There were no significant differences in the baseline characteristics between the two groups. The mean LV ejection fraction at baseline was $27.1 \pm 6.5 \%$, and most patients with NYHA class III or IV symptoms. Diagnostic angiography, biventricular angiography at baseline, and endomyocardial biopsy at baseline, 1 month, and 6 months were performed in all patients.

There was a significant improvement in the LVEF in the immunosuppressive therapy arm compared with placebo at 6 months: $88 \%$ of the patients in the treatment arm showed an improvement in LVEF $(26.4 \pm 6.9-48.0 \pm 7.3 \%)$ and LV end-diastolic diameter (LVEDD) $(68.6 \pm 7.4-52.8 \pm 6.3 \mathrm{~mm})$. None of the patients in the placebo arm improved, with even $83 \%$ of the patients deteriorating regarding LVEF $(27.6 \pm 6.6-19.5 \pm 4.8 \%)$ and LV dilatation $(69.2 \pm 7.9-75.3 \pm 7.4)$. 
In the immunosuppressive therapy arm, endomyocardial biopsy showed an improvement in inflammation, with disappearance of inflammatory infiltrates in patients who were classified as responders and in reduction or disappearance of inflammation with degeneration of myocytes in patients regarded as nonresponders. However, in biopsies of the placebo arm persistent myocardial inflammation and cell necrosis, with expansion of fibrosis, were detected.

The authors see their trial as the first randomized study in patients with proof of persistent inflammation and virus negative inflammatory cardiomyopathy in whom immunosuppression proved to be effective. However, they so far presented only changes in LVEF and LVEDD, while clinical outcomes remain to be determined. Larger multicenter studies are needed to confirm these interesting findings, since this could represent a novel approach to the management of heart failure in virus negative inflammatory cardiomyopathy.

\section{Blood pressure analyses from 25,595 patients (from 25,620 randomised) during the ONTARGET trial: reflections on current guidelines: $P$. Sleight (Oxford, Great Britain)}

The ONTARGET trial showed that telmisartan $(80 \mathrm{mg}$ per day) was "noninferior" to ramipril $(10 \mathrm{mg}$ per day) in 25,260 patients with coronary heart disease or diabetes plus additional risk factors who were over the age of 55 years of age but did not have evidence of heart failure [8].

The combination of the two drugs was associated with more adverse events without an increase in benefit. In a new observational analysis of the ONTARGET study the 25,260 patients were divided into four quartiles based upon blood pressure, regardless of which study arm they had been randomized to. The analysis showed that only the very highest quartile of BP (systolic BP $>154 \mathrm{~mm} \mathrm{Hg}$ ) had a significantly higher risk of the primary end point: cardiovascular death, stroke, MI, or heart-failure hospitalization $(P<0.001)$. When the end points were considered separately, there were no differences between the four BP quartiles for cardiovascular death or for MI. But those patients in the lowest quartile of BP (systolic $\leq 130 \mathrm{~mm} \mathrm{Hg}$ ) had significantly less risk of stroke than those in the highest quartile.

The investigators also reported about evidence of potential harm among diabetics: although those with diabetes in the highest quartile of blood pressure did have a higher risk of the primary outcome, when it came to cardiovascular death alone, those in the lowest quartile had an increased risk of death. The presenter suggested from these results, that in high risk people the guidelines of lowering blood pressure as much as possible may not apply.

\section{Telmisartan randomized assessment study in ACE-intolerant subjects with cardiovascular disease (TRANSCEND): K.K. Teo (Hamilton, ON, Canada)}

Angiotensin-converting enzyme (ACE) inhibitors reduce mortality and major cardiovascular events in patients with cardiovascular disease or high-risk diabetes, but are not tolerated by about $20 \%$ of patients, mainly due to cough but also due to hypotension, renal dysfunction, or angioneurotic edema. Angiotensin-receptor blockers (ARB) may be a valuable alternative in patients with or without heart failure that are intolerant to ACE inhibitors. Furthermore, some studies suggest that the combination of ACE inhibitors and ARBs is superior to their single use. Recently, the ONTARGET trial, that was designed as the biggest comparison to date of $A R B$ and ACE-inhibitor therapy or their combination in high-risk patients ( $>55$ years, coronary heart disease, peripheral artery disease, cerebrovascular disease, diabetes with endorgan damage) with controlled blood pressure and without evidence of heart failure, showed that the ARB telmisartan was noninferior to the ACE inhibitor ramipril in terms of blood-pressure-independent cardioprotection, and that the combination of the two did not offer any additional benefit $[8,9]$.

TRANSCEND, a sibling study to ONTARGET, was designed to assess whether the ARB telmisartan would be effective in high-risk patients with cardiovascular disease or diabetes with end-organ damage that are unable to tolerate ACE inhibitors. After a 3-week run-in period, 5,926 patients $>55$ years with coronary heart disease, peripheral artery disease, cerebrovascular disease, or high-risk diabetes without evidence of heart failure who were intolerant to ACE inhibitors were randomised to receive telmisartan $80 \mathrm{mg} /$ day or placebo, in addition to other usual therapies. Compared with similar trials, the patient population in TRANSCEND had more women, more patients with a history of stroke, and more with hypertension [10]. The primary outcome was the composite of cardiovascular death, myocardial infarction, stroke, or hospitalization for heart failure. Mean blood pressure was, on average, $4 \mathrm{mmHg}$ lower in the telmisartan group than in the placebo group throughout the study. After a median follow-up of 56 months, there was no difference in the composite primary end point, which occurred in $465(15.7 \%)$ of patients taking telmisartan and 504 $(17.0 \%)$ of those on placebo (HR $0.92 ; P=0.216$ ). 
Table 7 GISSI-HF trial

\begin{tabular}{|c|c|c|c|}
\hline End point & $\begin{array}{l}\text { Omega-3 fatty acids } \\
n=3,494(\%)\end{array}$ & $\begin{array}{l}\text { Placebo } \\
n=3,481 \text { (\%) }\end{array}$ & $\begin{array}{l}\text { Adjusted hazard } \\
\text { ratio }(95 \% \mathrm{Cl})\end{array}$ \\
\hline \multicolumn{4}{|l|}{ Primary end points } \\
\hline Mortality & 27.3 & 29.1 & $0.91(0.833-0.998)$ \\
\hline $\begin{array}{l}\text { All-cause mortality or hospitalization for } \\
\text { cardiovascular causes }\end{array}$ & 56.7 & 59.0 & $0.92(0.849-0.999)$ \\
\hline \multicolumn{4}{|l|}{ Secondary end points } \\
\hline Death from cardiovascular causes & 20.4 & 22.0 & $0.90(0.81-0.99)$ \\
\hline Sudden cardiac death & 8.8 & 9.3 & $0.93(0.79-1.08)$ \\
\hline Patients admitted for cardiovascular causes & 46.8 & 48.5 & $0.93(0.87-0.99)$ \\
\hline Patients with fatal and nonfatal Ml & 3.1 & 3.7 & $0.82(0.63-1.06)$ \\
\hline Patients with fatal and nonfatal stroke & 3.5 & 3.0 & $1.16(0.91-1.53)$ \\
\hline
\end{tabular}

Omega-3 fatty acid study: results of primary and secondary endpoints

One of the prespecified secondary outcomes, a composite of cardiovascular death, myocardial infarction, or stroke, occurred in $384(13.0 \%)$ patients on telmisartan compared with $440(14.8 \%)$ on placebo (HR 0.87, $P=0.048$ ). Eight hundred and ninety four $(30.3 \%)$ patients receiving telmisartan were hospitalized for a cardiovascular reason, compared with 980 (33.0\%) on placebo (RR 0.92; $P=0.025)$. One important aspect is that fewer patients discontinued study medication in the telmisartan group than in the placebo group, indicating that telmisartan was well tolerated in patients unable to tolerate ACE inhibitors. In summary, telmisartan had no significant effect on the primary endpoint of the study, and the preventive effect of the drug regarding cardiovascular death, myocardial infarction, or stroke, is modest at best.

The failure of telmisartan to reach the primary endpoint in TRANSCEND is rather surpising, particularly in light of the previous findings with $\mathrm{ACE}$ inhibitors from the HOPE, PEACE, and EUROPA trials. There are several potential explanations for the negative result of TRANSCEND that include a possible heterogeneity in the ARB class, but also the fact that TRANSCEND was ambitiously powered to find a $19 \%$ relative risk reduction [11]. A combined, timestratified analyses of TRANSCEND and PRoFESS [12] comparing telmisartan with placebo on outcome of cardiovascular death, myocardial infarction, stroke, and hospitalization for heart failure, showed no effect of telmisartan in the first 6 months, but a clear benefit after 6 months. These analyses suggest that there may be a delay of six to 12 months before the benefits of ARBs emerge. Based on ONTARGET and TRANSCEND, it seems reasonable that if the HOPE criteria apply, telmisartan or other ARBs may be used as an alternative to ACE inhibitors. The mechanisms behind the lack of effects on prevention of heart failure remain unknown. Therefore additional data are needed on ARBs, particularly in broader high-risk populations.

\section{Gruppo Italiano per lo Studio della Sopravvivenza nell'Infarto Miocardico Heart Failure (GISSI-HF): effect of $n-3$ polyunsaturated fatty acids in patients with chronic heart failure: L. Tavazzi (Pavia, Italy)}

Several epidemiological and experimental studies suggest that $n-3$ polyunsaturated fatty acids (PUFA) can exert favorable effects on atherothrombotic cardiovascular disease, including arrhythmias. GISSI-HF is a large-scale, randomized, double-blind, placebocontrolled study program consisting of two nested studies that were designed to investigate the effects of omega-3 fatty acids on one hand and statin therapy on the other hand on mortality and morbidity in patients with symptomatic heart failure.

In the PUFA study, investigators from 357 centers enrolled a total of 6,975 patients with CHF (NYHA class 2-4 irrespective of cause and LVEF), and randomized them to treatment with n-3 PUFA $1 \mathrm{~g}$ daily or placebo. Patients were followed up for a median of 3.9 years. The primary study endpoints were time to death, and time to death or admission to hospital for cardiovascular reasons. After 3.9 years of follow-up, treatment with the omega- 3 fatty acids reduced the RR of mortality by $9 \%$ and the RR of mortality and admission to the hospital for cardiovascular causes by $8 \%$ [13]. In absolute numbers, 955 (27.3\%) patients died from any cause in the n-3 PUFA group and 1,014 $(29.1 \%)$ in the placebo group (adjusted HR 0.91 [95.5\% CI 0.833-0.998], $P=0.041)$. Thousand nine hundred and eighty one (56.7\%) patients in the n-3 PUFA group and 2,053 (59.0\%) in the placebo group died or were admitted to hospital for cardiovascular reasons (adjusted HR 0.92 [99\% CI 0.849-0.999], $P=0.009$ ) (Table 7). There was no difference in the frequency of gastrointestinal disorders, representing the most common adverse reaction in both treatment groups [96 (3\%) n-3 PUFA group vs. 92 (3\%) placebo group]. While the absolute risk reduction for mortality was only $1.8 \%$, the number-needed-to-treat in 
order to avoid one death during the median follow-up of 3.9 years was 56, and the NNT to avoid one event like death or admission to hospital for cardiovascular reasons was 44. A per-protocol analysis that included only patients who remained on treatment for the study duration confirmed the overall findings, showing that treatment cut the absolute risk by 3.3\% compared with placebo, corresponding to a $14 \% \mathrm{RR}$ reduction. Although the overall benefit in patients with CHF was only modest, it should be noted that it was obtained in a population already treated with recommended therapies, and was consistent across all predefined subgroups.

Although the exact mechanisms of action remain unknown, omega-3 fatty acids could possibly exert favorable effects on inflammatory processes, such as reductions in endothelial activation and cytokine production, as well as influence platelet aggregation, blood pressure, heart rate, ventricular function, and autonomic tone. When compared to the GISSI Prevenzione trial $[14,15]$, in which n-3 PUFA supplementation reduced mortality by $21 \%$ in patients with MI mainly by influencing the risk of sudden death, this risk was not significantly different between the treated and untreated patients in GISSI-HF, suggesting that n-3 PUFA may act beneficially in heart-failure patients by other mechanisms of action than in postMI patients. Although questions remain about the mechanism of action, optimum dosing and formulation, supplementation with n-3 PUFA can provide a small beneficial advantage in terms of mortality and admission to hospital for cardiovascular reasons in CHF patients and therefore joins the short list of evidence-based life-prolonging therapies for heart failure [16].

\section{Gruppo Italiano per lo Studio della Sopravvivenza nell'Infarto Miocardico Heart Failure (GISSI-HF): effect of rosuvastatin in patients with chronic heart failure: Gianni Tognoni (Milan, Italy)}

Large observational studies, small prospective studies and post-hoc analyses of randomized clinical trials have suggested that HMG-CoA reductase inhibitors (statins) may exert beneficial effects in patients with CHF [17]. While these previous studies have been methodologically weak, the safety and efficacy of rosuvastatin in patients with heart failure have now been investigated in two placebo-controlled, randomized trials, the "Controlled Rosuvastatin in Multinational Trial in Heart Failure" (CORONA) [18], and the statin part of GISSI-HF [19].

Similar to the n-3 PUFA part of GISSI-HF (see above), the complementary study focussing on the effects of rosuvastatin was a randomised, double- blind, placebo-controlled trial that was conducted in 357 centers. Four thousand five hundred and seventy four patients with chronic heart failure (NYHA class 2-4, irrespective of cause and LVEF) were enrolled and randomly assigned to rosuvastatin $10 \mathrm{mg}$ or placebo. The primary endpoints were time to death, and time to death or admission to hospital for cardiovascular reasons. Treatment with rosuvastatin sufficiently decreased LDL cholesterol levels by $27 \%$ at 3 years from $123 \mathrm{mg} / \mathrm{dl}$ at baseline to $90 \mathrm{mg} / \mathrm{dl}$, and furthermore significantly decreased high-sensitivity $C$-reactive protein (CRP) levels. However, while omega-3 fatty-acid supplementation improved the morbidity and mortality in symptomatic heart failure patients in GISSI-HF, rosuvastatin failed to have any beneficial effect in the same group of patients. After a mean follow-up of 3.9 years, there was no significant difference between the treatment arms in either of the two co-primary end points: 657 (29\%) patients died from any cause in the rosuvastatin group and $644(28 \%)$ in the placebo group [adjusted HR 1.00 (95.5\% CI 0.90-1.12), $P=0.94]$. Thousand three hundred and five (57\%) patients in the rosuvastatin group and $1,283(56 \%)$ in the placebo group died or were admitted to hospital for cardiovascular reasons [adjusted HR 1.01 (99\% CI 0.91-1.11), $P=0.90$ ]. The findings were consistent across all secondary end points as well as consistent across every subgroup analyzed, including older patients, those with LVEF $>40 \%$, and those with and without diabetes. Hence, rosuvastatin $10 \mathrm{mg}$ daily did not affect clinical outcomes in patients with chronic heart failure of any cause, in whom the drug was safe.

The results of the GISSI-HF study are in line with findings from the CORONA trial. In CORONA, treatment with rosuvastatin had no significant effect on cardiovascular outcomes, as measured by the primary-end-point composite of cardiovascular death, nonfatal MI, or stroke. It did, however, significantly reduce the number of hospitalizations from cardiovascular causes and from heart failure [13]. Similarly, rosuvastatin did not reduce all-cause mortality and admission to the hospital for cardiovascular reasons in GISSI-HF [19], with patient's having less severe heart failure than in CORONA. Thus, both CORONA and GISSI-HF (rosuvastatin) are complementary and establish that, although statin therapy lowers the concentrations of LDL cholesterol, is well tolerated, and seems reasonably safe, it does not produce meaningful improvements in survival in patients with chronic heart failure. Therefore, the prescription of rosuvastatin or any statin to patients with heart failure should not be considered because the use of the cholesterol-lowering drugs does not translate into any clinical benefit for heart failure patients. 


\section{Arrhythmia}

\section{ATHENA: the effect of dronedarone on cardiovascular outcomes and stroke in patients with atrial fibrillation: S.J. Connolly (Hamilton, Canada)}

ATHENA is a placebo-controlled, double-blind, parallel arm trial to assess the efficacy of dronedarone $400 \mathrm{mg}$ two times a day versus placebo for the prevention of cardiovascular hospitalization or death from any cause in patients with AF or AFL.

A total of 4,628 patients were randomized from more than 550 sites in 37 countries: 2,301 to dronedarone and 2,327 to placebo for a minimum treatment and follow-up duration of 1 year.

About $42 \%$ of the patients were ages 75 years or older. Patients were stratified based on the presence or absence of AF or AFL at baseline, which was present in one-quarter of the patients randomized. Twelve percentage of the patients had evidence of systolic dysfunction, with an ejection fraction of lower than $45 \%$. About $30 \%$ of the patients had coexisting coronary artery disease. The rate of drug discontinuation was similar between the two arms as well as the rate of side effects.

Patients receiving dronedarone had-compared with placebo-a significant $24 \%$ reduction in hospitalizations or death due to cardiovascular causes over a follow-up time of about 21 months $(P<0.001)$. Compared with placebo, dronedarone induced a significant reduction in the risk of cardiovascular death by $30 \%(P=0.03)$. This was driven primarily by a significant $45 \%$ reduction in the incidence of death due to arrhythmias $(P=0.01)$. The overall mortality did not differ between the two arms (HR 0.84, $P=0.18)$. There was a significant reduction in the incidence of stroke in the dronedarone arm compared with placebo $(1.2 \%$ vs. $1.8 \%$, HR $0.66, P=0.027)$.

Although this trial did not directly compare dronedarone to amiodarone, indirect comparisons from this trial suggest that dronedarone may be similar in efficacy to amiodarone, without being associated with significant side effects. It will be therefore interesting to see the outcome of trials comparing dronedarone to amiodarone (e.g. DIONYSOS).

\section{Aortic stenosis}

Simvastatin plus ezetimibe in aortic stenosis: the SEAS study: T.R. Pedersen (Oslo, Norvay)

The SEAS study is the first large-scale randomised trial to assess the effects of lowering LDL-cholesterol in patients with aortic stenosis. The study included
1,873 patients with mild to moderate aortic stenosis without symptoms who were not considered to have a clear indication for treatment with cholesterol-lowering drugs [20]. Patients were randomly assigned to receive either intensive cholesterol lowering with the combination of simvastatin ( $40 \mathrm{mg}$ daily) and ezetimibe (10 $\mathrm{mg}$ daily) or matching placebo. The primary outcome was a composite of major cardiovascular events, including death from cardiovascular causes, aortic-valve replacement, nonfatal myocardial infarction, hospitalization for unstable angina pectoris, heart failure, coronary-artery bypass grafting, percutaneous coronary intervention, and nonhemorrhagic stroke. Secondary outcomes were events related to aortic-valve stenosis and ischemic cardiovascular events.

Compared with placebo, the combination of simvastatin and ezetimibe reduced LDL-cholesterol by an average of $61 \%$, corresponding to a reduction of about $2 \mathrm{mmol} / \mathrm{l}(76 \mathrm{mg} / \mathrm{dl})$, and this effect was sustained throughout the study. Six hundred and eighty eight patients had one or more primary endpoint events. No significant difference was observed between the treatment groups for the combined primary endpoint nor for the secondary endpoint of aortic valve disease events alone. The combination of simvastatin and ezetimibe did, however, produce a statistically significant $22 \%(P=0.02)$ proportional reduction in the secondary endpoint of atherosclerotic events alone: $15.7 \%$ in the simvastatin plus ezetimibe group versus $20.1 \%$ in the placebo group.

The study therapy was generally well tolerated. However, in the subsidiary safety analyses, a total of 175 patients were recorded with a serious adverse event attributed to cancer. More of these events were observed among patients assigned the combination of simvastatin and ezetimibe than among those assigned placebo [105 (11.1\%) vs. $70 \quad(7.5 \%)$; unadjusted $P=0.01]$. There were also slightly more cancer deaths [39 (4.1\%) vs. $23(2.5 \%)$; unadjusted $P=0.05$ ] (Table 8). These apparent differences were not related to any particular type of cancer and did not become significantly larger with more prolonged treatment.

The discussant of the study, Eugen Braunwald, explained, that the observed differences in cancer in the SEAS study are based on small numbers and could have occurred as a result of chance. In order to assess their relevance, the SEAS data have been provided to an independent academic group for combined analysis with data on cancer from the two other large trials of simvastatin and ezetimibe, which are still in progress [21]. The SHARP (Study of Heart and Renal Protection) study is a randomized placebo-controlled trial of simvastatin and ezetimibe in 9,400 patients with chronic kidney disease. The IMPROVE-IT (IMProved Reduction of Outcomes: Vytorin Efficacy 
Table 8 SEAS: any fatal or nonfatal cancer and death from cancer (Oxford analysis)

\begin{tabular}{llll}
\hline Value & $\begin{array}{l}\text { Active treatment } \\
(n=944)\end{array}$ & $\begin{array}{l}\text { Control } \\
(n=929)\end{array}$ & $\begin{array}{l}P \\
\text { (unadjusted) }\end{array}$ \\
\hline $\begin{array}{l}\text { Any fatal or nonfatal } \\
\text { cancer }(n)\end{array}$ & 101 & 65 & 0.006 \\
$\begin{array}{l}\text { Percent per year } \\
\text { Death from any cancer } \\
(n)\end{array}$ & 2.7 & 1.7 & - \\
$\begin{array}{l}\text { Percent per year } \\
\text { Percent }\end{array}$ & 0.9 & 20 & 0.04 \\
\hline
\end{tabular}

International Trial) study is a randomized doubleblind trial of simvastatin and ezetimibe compared to simvastatin alone which has recruited 12,000 of a planned 18,000 patients with acute coronary disease. In combination, the SHARP and IMPROVE-IT studies involve about four times as many cancers as in the SEAS study. Independent analysis of these data with was initiated and has been conducted and interpreted by the Clinical Trial Service Unit at the University of Oxford, UK. Their analysis of SHARP and IMPROVEIT does so far not support the suggestion of an increase in cancer that was raised by the subsidiary analyses of the relatively small numbers of cancers in the SEAS study [21] (Table 9). However the follow up time of SHARP and IMPROVE-IT are at the time
Table 9 IMPROVE-IT and SHARP: any fatal or nonfatal cancer and death from cancer (Oxford analysis)

\begin{tabular}{llll}
\hline Value & $\begin{array}{l}\text { Active treatment } \\
(n=10,319)\end{array}$ & $\begin{array}{l}\text { Control } \\
(n=10,298)\end{array}$ & $\begin{array}{l}P \\
\text { (unadjusted) }\end{array}$ \\
\hline $\begin{array}{l}\text { Any fatal or nonfatal } \\
\quad \text { cancer }(n)\end{array}$ & 313 & 326 & 0.61 \\
$\begin{array}{l}\text { Percent per year } \\
\text { Death from any } \\
\quad \text { cancer }(n)\end{array}$ & 1.7 & 1.8 & - \\
Percent per year & 97 & 72 & 0.07 \\
\hline
\end{tabular}

being much shorter than in SEAS, so the final data have to be carefully watched [22].

In conclusion, the SEAS study has found that intensive LDL-cholesterol lowering with the combination of simvastatin and ezetimibe in patients with mild to moderate aortic stenosis does appear to reduce the risk of coronary artery disease events (as has been shown for many other types of patient in previous trials) but not the rate of progression of aortic valve disease. The use of simvastatin and ezetimibe in such patients was generally well tolerated and safe. However due to the increase in cancer rates, the final results of the SHARP and IMPROVE-IT trials have to be evaluated for cancer risk and cancer death.

\section{References}

1. Stone GW, Witzenbichler B, Guagliumi G, Peruga JZ, Brodie BR, Dudek D, Kornowski R, Hartmann F, Gersh BJ, Pocock SJ, Dangas G, Wong SC, Kirtane AJ, Parise H, Mehran R, HORIZONS-AMI Trial Investigators (2008) Bivalirudin during primary PCI in acute myocardial infarction. $\mathrm{N}$ Engl J Med 358:2218-2230

2. Serruys PW, Garcia-Garcia HM, Buszman P, Erne P, Verheye S, Aschermann M, Duckers H, Bleie O, Dudek D, Botker HE, von Birgelen C, D'Amico D, Hutchinson T, Zambanini A, Mastik F, van Es GA, van der Steen AF, Vince DG, Ganz P, Hamm CW, Wijns W, Zalewski A (2008) Effects of the direct lipoprotein-associated phospholipase A2 inhibitor darapladib on human coronary atherosclerotic plaque. Circulation 118:1172-1182

3. Fox K, Ford I, Steg PG, Tendera M, Ferrari R (2008) Ivabradine for patients with stable coronary artery disease and left-ventricular systolic dysfunction (BEAUTIFUL): a randomised, doubleblind, placebo-controlled trial. Lancet 372:807-816
4. Reil JC, Böhm M (2007) The role of heart rate in the development of cardiovascular disease. Clin Res Cardiol 96:585-592

5. Fox K, Ford I, Steg PG, Tendera M, Robertson M, Ferrari R (2008) Heart rate as a prognostic risk factor in patients with coronary artery disease and left-ventricular systolic dysfunction (BEAUTIFUL): a subgroup analysis of a randomised controlled trial. Lancet 372:817-821

6. Reil JC, Böhm M (2008) BEAUTIFUL results-the slower, the better? Lancet 372:779-780

7. Brunner-La Rocca HP, Buser PT, Schindler R, Bernheim A, Rickenbacher P, Pfisterer M (2006) Management of elderly patients with congestive heart failure-design of the trial of intensified versus standard medical therapy in elderly patients with congestive heart failure (TIME-CHF). Am Heart J 151:949-955
8. Yusuf S, Teo KK, Pogue J, Dyal L, Copland I, Schumacher H, Dagenais G, Sleight P, Anderson C (2008) Telmisartan, ramipril, or both in patients at high risk for vascular events. $\mathrm{N}$ Engl J Med 358:1547-1559

9. Clever YP, Rosenkranz S, Böhm M, Scheller B (2008) Hotline update of clinical trials and registries presented at the ACC and SCAI-ACCi2 meeting 2008 in Chicago. Clin Res Cardiol 97:409-417

10. The TRANSCEND Investigators (2008) Effects of the angiotensin-receptor blocker telmisartan on cardiovascular events in high-risk patients intolerant to angiotensin-converting enzyme inhibitors: a randomized controlled trial. Lancet. doi:10.1016/SO1406736(08)61242-8

11. Ripley TR, Harrison D (2008) The power to TRANSCEND. Lancet. doi:10.1016/SO140-6736(08)61243-X

12. Yusuf S, Diener HC, Sacco RL et al. for the PRoFESS Study Group (2008) Telmisartan to prevent recurrent stroke and cardiovascular events. $\mathrm{N}$ Engl J Med 359. doi:10.1056/NEJMoa0804593 
13. GISSI-HF Investigators (2008) Effect of n-3 polyunsaturated fatty acids in patients with chronic heart failure (the GISSI-HF trial): a randomized, doubleblind, placebo-controlled trial. Lancet. doi:10.1016/S0140-6736(08)61241-6.

14. GISSI-Prevenzione Investigators (1999) Dietary supplementation with $\mathrm{n}-3$ polyunsaturated fatty acids and vitamin $\mathrm{E}$ after myocardial infarction: results of the GISSI-Prevenzione trial. Gruppo Italiano per lo Studio della Sopravvivenza nell'Infarto miocardico. Lancet 354:447-455

15. Marchioli R, Barzi F, Bomba E, Chieffo C, Di Gregorio D, Di Mascio R, Franzosi MG, Geraci E, Levantesi G, Maggioni AP, Mantini L, Marfisi RM, Mastrogiuseppe G, Mininni N, Nicolosi GL, Santini M, Schweiger C, Tavazzi L, Tognoni G, Tucci C, Valagussa F (2002) Early protection against sudden death by $n-3$ polyunsaturated fatty acids after myocardial infarction: time-course analysis of the results of the Gruppo Italiano per lo Studio della Sopravvivenza nell'Infarto Miocardico (GISSI)Prevenzione. Circulation 105:18971903

16. Fonarow GC (2008) Statins and n-3 fatty acid supplementation in heart failure. Lancet. doi:10.1016/S01406736(08)61239-8.

17. van der Harst $P$, Böhm M, van Gilst WH, van Veldhuisen DJ (2008) The case for statin therapy in chronic heart failure. Clin Res Cardiol 97:139-146

18. Kjekshus J, Apetrei E, Barrios V, Böhm M, Cleland JG, Cornel JH, Dunselman P, Fonseca C, Goudev A, Grande P, Gullestad L, Hjalmarson A, Hradec J, Janosi A, Kamensky G, Komajda M, Korewicki J, Kuusi T, Mach F, Mareev V, McMurray JJ, Ranjith N, Schaufelberger M, Vanhaecke J, van Veldhuisen DJ, Waagstein F, Wedel H, Wikstrand J (2007) Rosuvastatin in older patients with systolic heart failure. N Engl J Med 357:2248-2261
19. GISSI-HF investigators (2008) Effect of rosuvastatin in patients with chronic heart failure (the GISSI-HF trial): a randomized, double-blind, placebocontrolled trial. Lancet. doi:10.1016/ S0140-6736(08)61241-6.

20. Rossebo AB, Pedersen TR, Boman K, Brudi P, Chambers JB, Egstrup K, Gerdts E, Gohlke-Bärwolf C, Holme I, Kesaniemi YA, Malbecq W, Nienaber CA, Ray S, Skjaerpe T, Wachtell K, Willenheimer R (2008) Intensive lipid lowering with simvastatin and ezetimibe in aortic stenosis. N Engl J Med [Epub ahead of print 2 Sep]

21. Peto R, Emberson J, Landray M, Baigent $C$, Collins $R$, Clare $R$, Califf $R$ (2008) Analyses of cancer data from three ezetimibe trials. N Engl J Med [Epub ahead of print $2 \mathrm{Sep}$ ]

22. Drazen JM, D'Agostino RB, Ware JH, Morrissey S, Curfman GD (2008) Ezetimibe and cancer-an uncertain association. N Engl J Med [Epub ahead of print 2 Sep] 